\title{
Trial sequential analysis in meta-analyses: A clinically oriented approach with real-world example
}

Jeffrey Shi Kai Chan, MBChB, ${ }^{a, b}$ and Amer Harky, MBChB, MRCS, MSc ${ }^{c}$

Supplemental material is available online.

Ranked high on the hierarchy of evidence, meta-analyses are important summaries of the existing literature, often influencing clinical practice and guidelines. However, as evidence accumulates, random errors may at times lead to spuriously significant results, and an increasing frequency of statistical testing by meta-analyses increases the likelihood of such being reported; that is, type 1 error. ${ }^{1}$ Therefore, trial sequential analysis (TSA) has been developed to adjust for this increase in type 1 error. TSA is conceptually similar to sequential interim analyses of randomized controlled trials, where trial results are tested at regular intervals to determine whether a certain difference due to the intervention, or the lack thereof, has been conclusively demonstrated by existing data, allowing the trial to be terminated. ${ }^{2,3}$

In TSA of meta-analyses, data from each study is analogous to data added during each sequential interim analysis. Statistical techniques are used to adjust and increase the threshold for statistical significance (z-score threshold; conventionally corresponds to a $P$ value of .05 ) based on the effect to be observed, incidence of outcome in the control arm, information size (a statistical measure of the amount of data analyzed), and heterogeneity. Simply put, a large effect (eg, 90\% relative risk reduction [RRR]) observed from data with minimal heterogeneity requires smaller information sizes for the observation to be conclusive than a small effect (eg, 10\% RRR) observed from data with significant heterogeneity. As such, TSA may be used to determine whether certain effects (ie, differences between 2 arms, such as a $25 \%$ RRR) may be considered conclusive in

\footnotetext{
From the a Faculty of Medicine, The Chinese University of Hong Kong, Shatin, New Territories, Hong Kong; ${ }^{b}$ Division of Cardiology, Department of Medicine and Therapeutics, Prince of Wales Hospital, Shatin, New Territories, Hong Kong; and ${ }^{\mathrm{c}}$ Department of Cardiothoracic Surgery, Liverpool Heart and Chest Hospital, Liverpool, United Kingdom.

Received for publication March 23, 2020; revisions received June 6, 2020; accepted for publication June 10, 2020; available ahead of print June 27, 2020.

Address for reprints: Jeffrey Shi Kai Chan, MBChB, Faculty of Medicine, The Chinese University of Hong Kong, Shatin, New Territories, Hong Kong (E-mail: skjchan@link.cuhk.edu.hk).

J Thorac Cardiovasc Surg 2021;162:167-73

$0022-5223 / \$ 36.00$

Copyright (C) 2020 by The American Association for Thoracic Surgery

https://doi.org/10.1016/j.jtcvs.2020.06.063
}

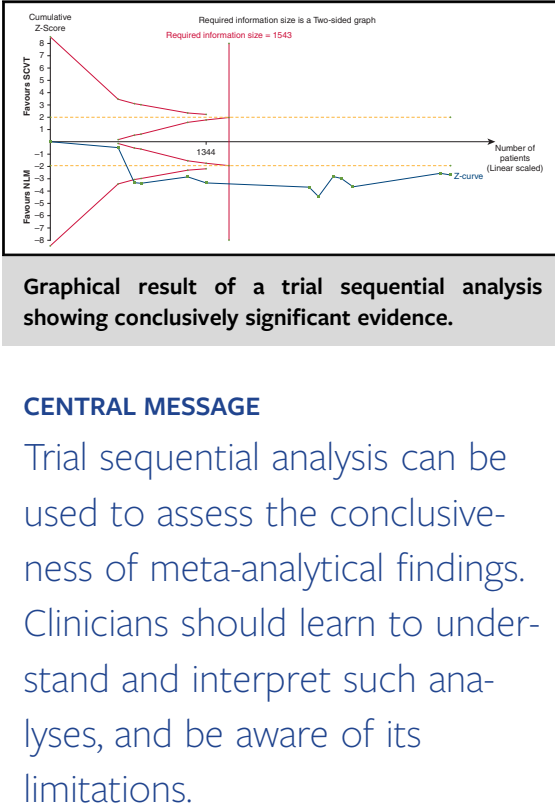

See Commentaries on pages 174,175 , and 176.

a meta-analysis. Results found to be conclusive may not require much further investigation, whereas premature and spurious conclusions based on unadjusted significant results can also be avoided.

Meta-analyses with $\mathrm{TSA}^{4-8}$ and post hoc $\mathrm{TSA}^{9-12}$ have become more common in recent years, having been incorporated in some Cochrane reviews as well. ${ }^{8}$ It is therefore important for clinicians to understand the principles, interpretation, and limitations of TSA. In this article, we aim to illustrate these with a post hoc TSA using data from a meta-analysis by Woldendorp and colleagues. ${ }^{13}$ The meta-analysis shows an important association between subclinical valve thrombosis (SCVT) of transcatheter aortic valve implantation and stroke, and the superiority of oral anticoagulants (OAC) to single (SAPT) or dual (DAPT) antiplatelet therapy in preventing SCVT.

To perform the TSA, study-level summary data of all meta-analyzed comparisons were obtained from the published Forrest plots, with odds ratios as summary estimates in accordance with the choice by Woldendorp and colleagues. ${ }^{13}$ Single-arm studies were excluded. Two-sided $z$-score thresholds were adjusted using the O'BrienFleming $\alpha$-spending function with 2 -sided $5 \%$ type 1 error 
and $80 \%$ power. The DerSimonian-Laird random effect model was used. Control arm incidences were calculated using event rates from all studies. Information sizes were estimated from all available statistical information (Fischer information). If that was impossible, sample sizes were used instead. In such cases, information size estimations were repeated using incidences of outcome event (event sizes) to ensure the robustness of calculations. RRR values were estimated from all studies. ${ }^{14}$ To demonstrate the interpretation and utility of TSA performed using prespecified RRR values, TSA was also repeated with RRR values deemed appropriate for the comparison made.
Studies reporting no event were handled by adding a constant (1) to both the event and sample counts. All statistical analyses were performed using TSA software version 0.9.5.10 Beta, Copenhagen trial unit. ${ }^{14}$ All TSA data files are available online (Online Data Supplement 1-3).

TSA generates graphical outputs (Figures 1-4). The $y$-axis represents the cumulative $z$-score of included studies, which is a measure of the statistical strength of the cumulative evidence, whereas the $x$-axis represents information size. The blue line is the $z$-score curve, which is a plot of the cumulative $z$-score with study data added and plotted chronologically. These data points may

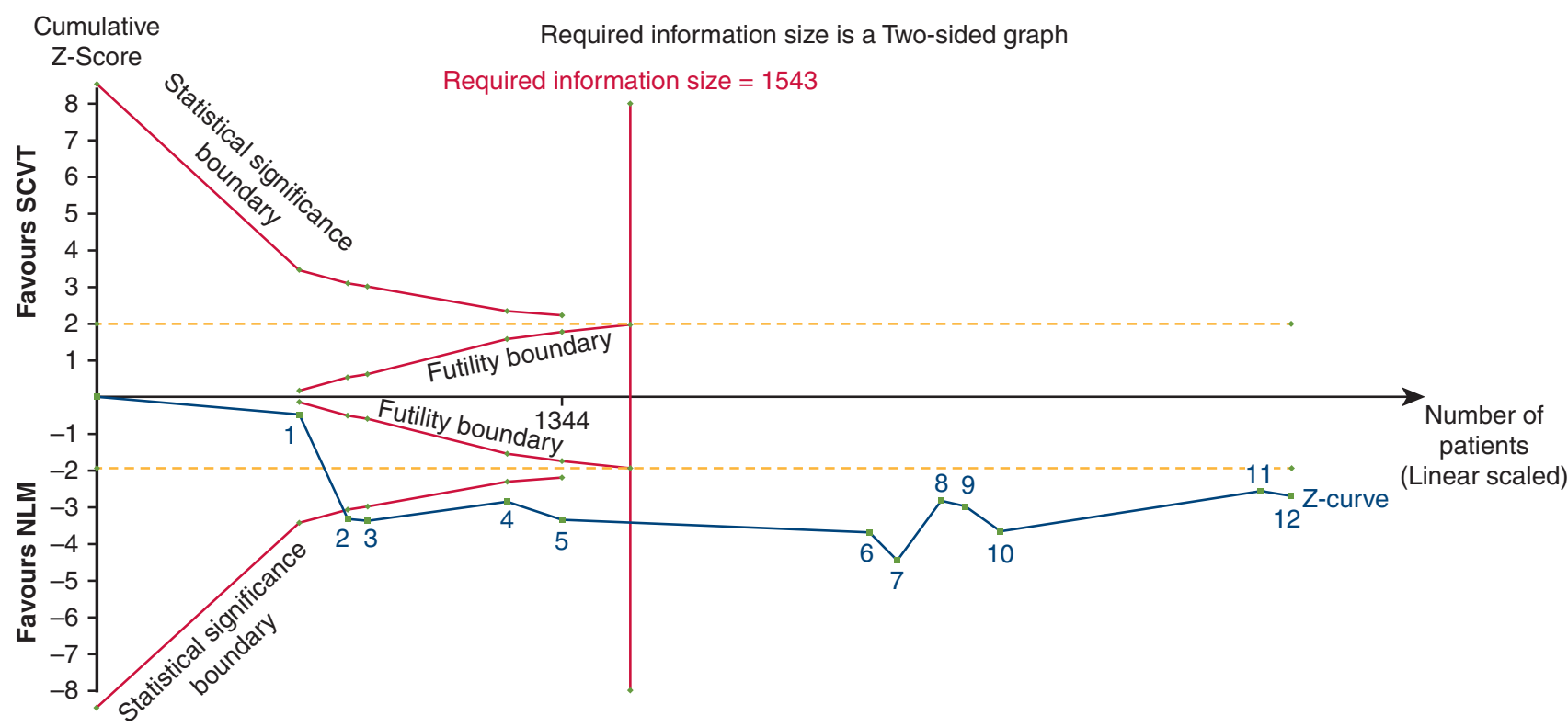

FIGURE 1. Result of Trial Sequential Analysis comparing rates of stroke in patients with subclinical valvular thrombosis (SCVT) and normal leaflet motions $(N L M)$. Here, the $z$-score curve (blue line) crossed both the required information size (vertical red line) and the conventional statistical significance boundary corresponding to 2 -sided $P$ value of .05 (dotted yellow lines). Such indicates that the observed increase in stroke rate in patients in SCVT could be considered conclusive with the existing evidence. The numbers on the blue line indicate the chronologically arranged set of studies, from which the data were included in this analysis: 1: Erungaren JJ, Sulimov DS, El-Mawardy M, Sato T, Allali A, Landt M, et. al. TCT-99 valve thrombosis after transcatheter aortic valve replacement: incidence, characteristics and outcomes. J Am Coll Cardiol. 2015;66:B46, 2: Leetmaa T, Hansson NC, Leipsic J, Jensen K, Poulsen SH, Andersen HR, et. al. Early aortic transcatheter heart valve thrombosis. Circ Cardiovasc Interv. 2015;8:e001596, 3: Makkar RR, Fontana G, Jilaihawi H, Chakravarty T, Kofoed KF, de Backer O, et. al. Possible subclinical leaflet thrombosis in bioprosthetic aortic valves. N Engl J Med. 2015;373:2015-24, 4: Hansson NC, Grove EL, Andersen HR, Leipsic J, Mathiassen ON, Jensen JM, et. al. Transcatheter aortic valve thrombosis. J Am Coll Cardiol. 2016;68:2059-69, 5: Pache G, Schoechlin S, Blanke P, Dorfs S, Jander N, Arepalli CD, et. al. Early hypo-attenuated leaflet thickening in balloon-expandable transcatheter aortic heart valves. Eur Heart J. 2016;37:2263-71, 6: Chakravarty T, Søndergaard L, Friedman J, de Backer O, Berman D, Kofoed KF, et. al. Subclinical leaflet thrombosis in surgical and transcatheter bioprosthetic aortic valves: an observational study. Lancet 2017;389:2383-92, 7: Marwan M, Mekkhala N, Göller M, Röther J, Bittner D, Schuhbaeck A, et. al. Leaflet thrombosis following transcatheter aortic valve implantation. J Cardiovasc Comput Tomogr. 2018;12:8-13, 8: Vollema EM, Kong WKF, Katsanos S, Kamperidis V, van Rosendael PJ, van der Kley F, et. al. Transcatheter aortic valve thrombosis: the relation between hypo-attenuated leaflet thickening, abnormal valve haemodynamics, and stroke. Eur Heart J. 2017;38:1207-17, 9: Yanagisawa R, Hayashida K, Yamada Y, Tanaka M, Yashima F, Inohara T, et. al. Incidence, predictors, and mid-term outcomes of possible leaflet thrombosis after TAVR. J Am Coll Cardiol Imaging. 2017;10:1-11, 10: Basra SS, Gopal A, Hebeler KR, Baumgarten H, Anderson A, Potluri SP, et. al. Clinical leaflet thrombosis in transcatheter and surgical bioprosthetic aortic valves by four-dimensional computed tomography. Ann Thorac Surg. 2018;106:1716-25, 11: Ruile P, Minners J, Breitbart P, Schoechlin S, Gick M, Pache G, et. al. Medium-term follow-up of early leaflet thrombosis after transcatheter aortic valve replacement. J Am Coll Cardiol Interv. 2018;11:1164-71, and 12: Jimenez C, Ohana M, Marchandot B, Kibler M, Carmona A, Peillex M, et. al. Impact of antithrombotic regimen and platelet inhibition extent on leaflet thrombosis detected by cardiac MDCT after transcatheter aortic valve replacement. J Clin Med. 2019;8:506-17. 


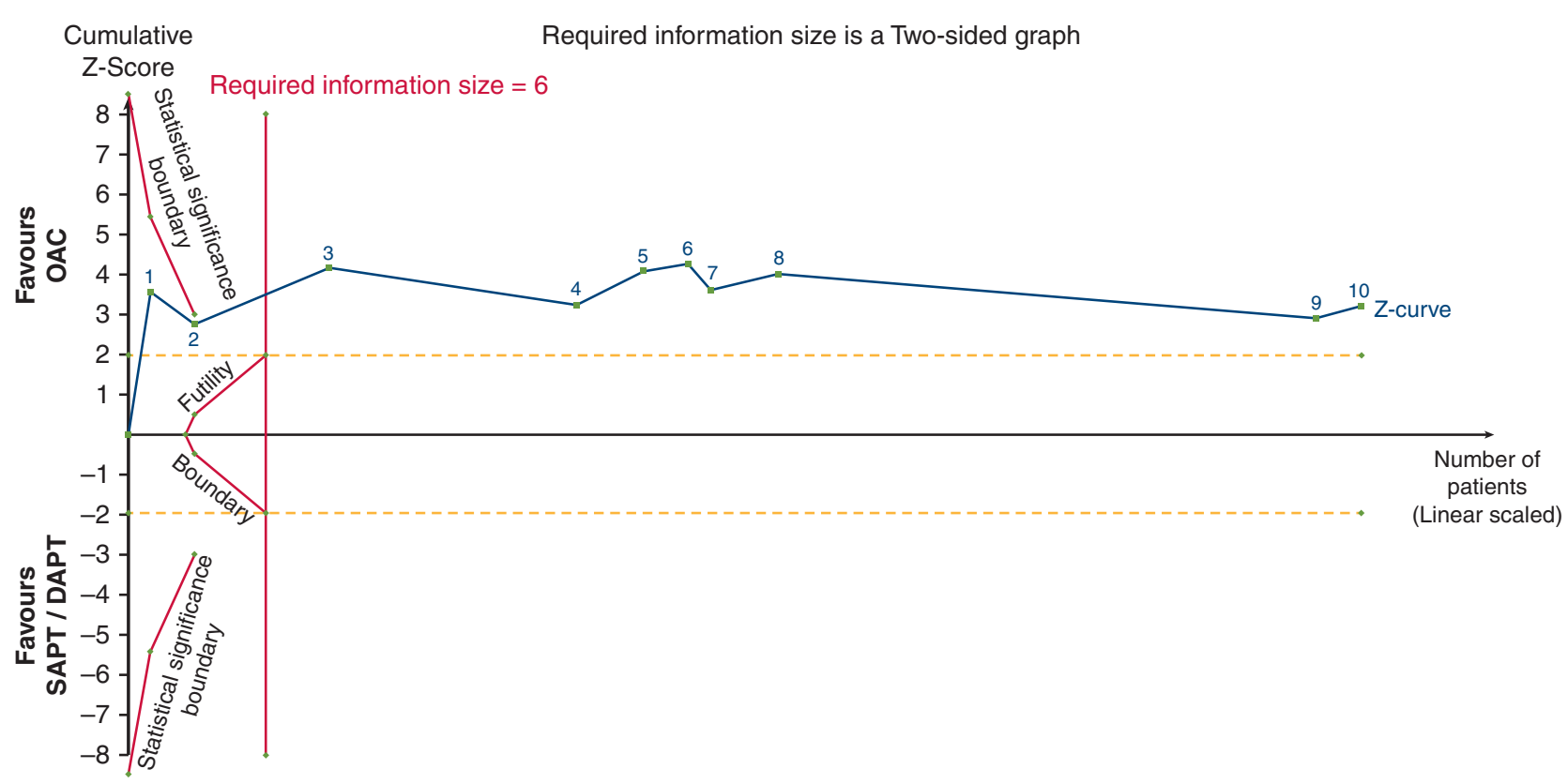

FIGURE 2. Result of Trial Sequential Analysis comparing rates of subclinical valvular thrombosis in patients taking oral anticoagulants $(O A C)$ versus single antiplatelet therapy (SAPT) or dual antiplatelet therapy (DAPT). Here, the $z$-score curve (blue line) crossed both the required information size (vertical red line) and the conventional statistical significance boundary corresponding to 2 -sided $P$ value of .05 (dotted yellow lines). Such indicates that the observed reduction in rate of subclinical valvular thrombosis in patients taking OAC could be considered conclusive with the existing evidence. The numbers on the blue line indicate the chronologically arranged set of studies, from which the data were included in this analysis: 1: Leetmaa T, Hansson NC, Leipsic J, Jensen K, Poulsen SH, Andersen HR, et al. Early aortic transcatheter heart valve thrombosis. Circ Cardiovasc Interv. 2015;8:e001596, 2: Makkar RR, Fontana G, Jilaihawi H, Chakravarty T, Kofoed KF, de Backer O, et al. Possible subclinical leaflet thrombosis in bioprosthetic aortic valves. $N$ Engl J Med. 2015;373:2015-24, 3: Hansson NC, Grove EL, Andersen HR, Leipsic J, Mathiassen ON, Jensen JM, et al. Transcatheter aortic valve thrombosis. J Am Coll Cardiol. 2016;68:2059-69, 4: Chakravarty T, Søndergaard L, Friedman J, de Backer O, Berman D, Kofoed KF, et al. Subclinical leaflet thrombosis in surgical and transcatheter bioprosthetic aortic valves: an observational study. Lancet. 2017;389:2383-92, 5: Marwan M, Mekkhala N, Göller M, Röther J, Bittner D, Schuhbaeck A, et al Leaflet thrombosis following transcatheter aortic valve implantation. J Cardiovasc Comput Tomogr. 2018;12:8-13, 6: Vollema EM, Kong WKF, Katsanos S, Kamperidis V, van Rosendael PJ, van der Kley F, et al. Transcatheter aortic valve thrombosis: the relation between hypo-attenuated leaflet thickening, abnormal valve haemodynamics, and stroke. Eur Heart J. 2017;38:1207-17, 7: Yanagisawa R, Hayashida K, Yamada Y, Tanaka M, Yashima F, Inohara T, et al. Incidence, predictors, and mid-term outcomes of possible leaflet thrombosis after TAVR. J Am Coll Cardiol Imaging 2017;10:1-11, 8: Basra SS, Gopal A, Hebeler KR, Baumgarten H, Anderson A, Potluri SP, et al. Clinical leaflet thrombosis in transcatheter and surgical bioprosthetic aortic valves by four-dimensional computed tomography. Ann Thorac Surg. 2018;106:1716-25, 9: Ruile P, Minners J, Breitbart P, Schoechlin S, Gick M, Pache G, et al. Medium-term follow-up of early leaflet thrombosis after transcatheter aortic valve replacement. J Am Coll Cardiol Interv. 2018;11:1164-71, and 10: Jimenez C, Ohana M, Marchandot B, Kibler M, Carmona A, Peillex M, et al. Impact of antithrombotic regimen and platelet inhibition extent on leaflet thrombosis detected by cardiac MDCT after transcatheter aortic valve replacement. J Clin Med. 2019;8:506-17.

represent the sample size, event size, or a combination of both of these and the number of trials (also called Fischer information), depending on the specification of the analysis. Generally, Fischer information is preferred and superior to the other options. The brown, dotted lines represent the conventional statistical significance threshold for 2 -sided $z$-score values of \pm 1.96 (these are optional and not always present in TSA plots). The red lines represent the adjusted boundaries (Figure 1) - a pair of outer, oblique red lines form the statistical significance boundaries, whereas the inner oblique lines form the futility boundaries. Crossing either of the statistical significance boundaries imply that the existing, statistically significant data are conclusive; similarly, crossing either of the futility boundaries imply that the existing, statistically insignificant data are conclusive. The final vertical red line represents the required information size (RIS) — upon reaching this information size, all observations are said to be stable because effects are unlikely to fluctuate significantly even upon addition of new data, and a conclusion has therefore likely been reached. The statistical significance boundaries become narrower and approaches the conventional statistical significance threshold as information sizes increases, reflecting that the significance threshold is penalized and adjusted more heavily when the information size is smaller.

Figure 1 shows the TSA results comparing stroke rates in patients with SCVT or normal leaflet motions (NLM) (stroke rate in the latter, $2.75 \%$ ). Sample sizes were used 


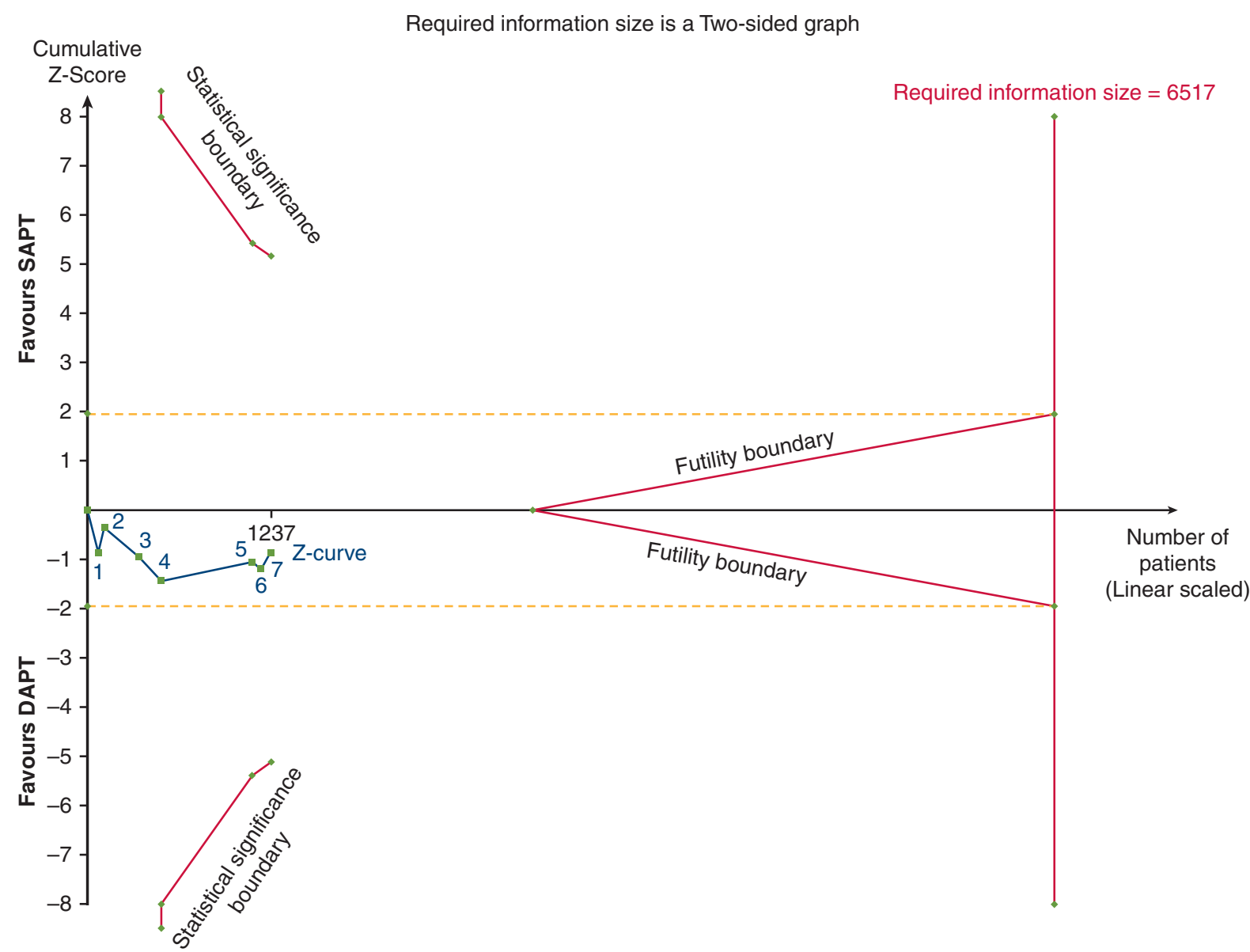

FIGURE 3. Result of trial sequential analysis comparing rates of subclinical valvular thrombosis in patients taking single antiplatelet therapy (SAPT) versus dual antiplatelet therapy (DAPT). Here, the $z$-score curve (blue line) did not cross any boundary (red lines). Such indicates that the observed difference in rates of subclinical valvular thrombosis between the 2 arms could not be considered conclusive with the existing evidence. The numbers on the blue line indicate the chronologically arranged set of studies, from which the data were included in this analysis: 1: Leetmaa T, Hansson NC, Leipsic J, Jensen K, Poulsen SH, Andersen HR, et al. Early aortic transcatheter heart valve thrombosis. Circ Cardiovasc Interv. 2015;8:e001596, 2: Makkar RR, Fontana G, Jilaihawi H, Chakravarty T, Kofoed KF, de Backer O, et al. Possible subclinical leaflet thrombosis in bioprosthetic aortic valves. N Engl J Med 2015;373:2015-24, 3: Hansson NC, Grove EL, Andersen HR, Leipsic J, Mathiassen ON, Jensen JM, et al. Transcatheter aortic valve thrombosis. J Am Coll Cardiol. 2016;68:2059-69, 4: Pache G, Schoechlin S, Blanke P, Dorfs S, Jander N, Arepalli CD, et al. Early hypo-attenuated leaflet thickening in balloon-expandable transcatheter aortic heart valves. Eur Heart J. 2016;37:2263-71, 5: Chakravarty T, Søndergaard L, Friedman J, de Backer O, Berman D, Kofoed KF, et al. Subclinical leaflet thrombosis in surgical and transcatheter bioprosthetic aortic valves: an observational study. Lancet 2017;389:2383-92, 6: Yanagisawa R, Hayashida K, Yamada Y, Tanaka M, Yashima F, Inohara T, et al. Incidence, predictors, and mid-term outcomes of possible leaflet thrombosis after TAVR. J Am Coll Cardiol Imaging. 2017;10:1-11, and 7: Basra SS, Gopal A, Hebeler KR, Baumgarten H, Anderson A, Potluri SP, et al. Clinical leaflet thrombosis in transcatheter and surgical bioprosthetic aortic valves by four-dimensional computed tomography. Ann Thorac Surg. 2018;106:1716-25.

to estimate information sizes as Fischer information could not be used due to too little information use $(-141.9 \%)$. In Figure 1, the $z$-score curve crossed the RIS, indicating that the observed effect, that is, stroke occurring significantly more frequently in patients with SCVT, is stable and conclusive. With an observed RRR of $-188 \%$, the TSA was repeated with a prespecified RRR of $-100 \%$. This revealed the $z$-score curve crossing the statistical significance boundary, indicating that the existing evidence was sufficient to reach a conclusion that there is at least a $100 \%$ increase in risk of stroke in patients with SCVT compared with those with NLM. This was because more information is required to conclude a smaller effect size. So, given that the current evidence is sufficient to conclusively demonstrate a $100 \%$ increase in risk of stroke, it is also sufficient to conclusively demonstrate larger effect 


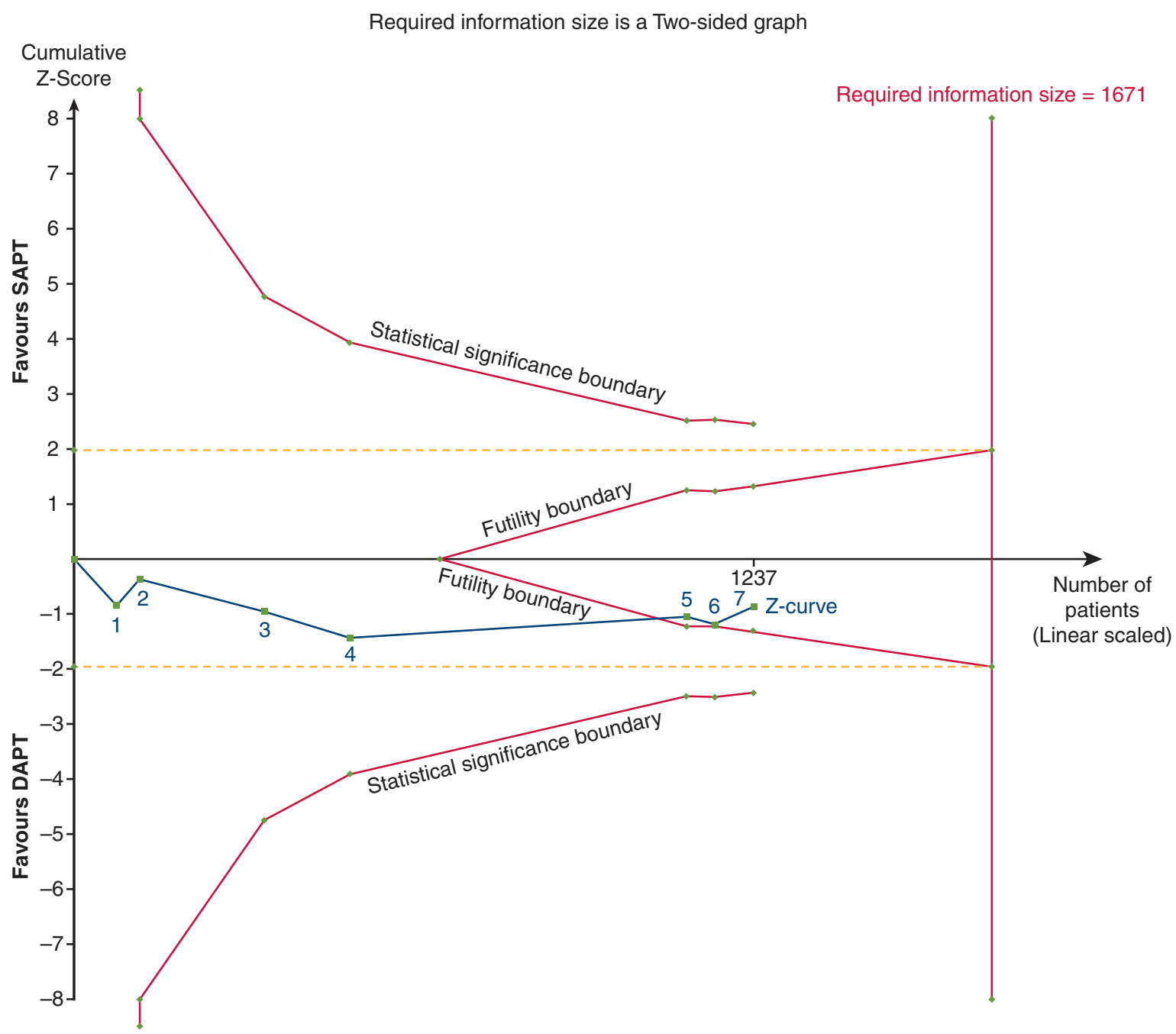

FIGURE 4. Result of trial sequential analysis comparing rates of subclinical valvular thrombosis (SCVT) in patients taking single antiplatelet therapy $(S A P T)$ versus dual antiplatelet therapy (DAPT), with required information sizes estimated with a $-33.3 \%$ relative risk reduction. Here, the $z$-score curve (blue line) crossed the futility boundary (red lines). Such indicates that the existing evidence is sufficient to conclude that SAPT usage is not associated with an increase in SCVT risk of $33.3 \%$ or more compared to DAPT. The numbers on the blue line indicate the chronologically arranged set of studies, from which the data were included in this analysis: 1: Leetmaa T, Hansson NC, Leipsic J, Jensen K, Poulsen SH, Andersen HR, et al. Early aortic transcatheter heart valve thrombosis. Circ Cardiovasc Interv. 2015;8:e01596, 2: Makkar RR, Fontana G, Jilaihawi H, Chakravarty T, Kofoed KF, de Backer O, et al. Possible subclinical leaflet thrombosis in bioprosthetic aortic valves. N Engl J Med. 2015;373:2015-24, 3: Hansson NC, Grove EL, Andersen HR, Leipsic J, Mathiassen ON, Jensen JM, et al. Transcatheter aortic valve thrombosis. J Am Coll Cardiol. 2016;68:2059-206, 4: Pache G, Schoechlin S, Blanke P, Dorfs S, Jander N, Arepalli CD, et. al. Early hypo-attenuated leaflet thickening in balloon-expandable transcatheter aortic heart valves. Eur Heart J. 2016;37:226371, 5: Chakravarty T, Søndergaard L, Friedman J, de Backer O, Berman D, Kofoed KF, et. al. Subclinical leaflet thrombosis in surgical and transcatheter bioprosthetic aortic valves: an observational study. Lancet. 2017;389:2383-92, 6: Yanagisawa R, Hayashida K, Yamada Y, Tanaka M, Yashima F, Inohara T, et. al. Incidence, predictors, and mid-term outcomes of possible leaflet thrombosis after TAVR. J Am Coll Cardiol Imaging. 2017;10:1-11, and 7: Basra SS, Gopal A, Hebeler KR, Baumgarten H, Anderson A, Potluri SP, et. al. Clinical leaflet thrombosis in transcatheter and surgical bioprosthetic aortic valves by four-dimensional computed tomography. Ann Thorac Surg. 2018;106:1716-25.

sizes. Similar observations were made when the TSA was performed using event size instead, suggesting that the calculation was robust.

Figure 2 shows the TSA results comparing OAC to SAPT or DAPT for preventing SCVT (SAPT or DAPT arm incidence, $18.71 \%$ ). Again, the $z$-score curve crossed the RIS, suggesting that the observed effects could be considered conclusive. With an observed RRR of $68.6 \%$, the TSA was repeated with an RRR of $20 \%$. Similarly, the $z$-score curve crossed the RIS, indicating that the existing evidence is sufficient to conclude that there is at least a $20 \%$ reduction in SCVT incidence in 
patients taking OAC, compared with those taking SAPT or DAPT.

Figure 3 shows the TSA results comparing SAPT to DAPT for preventing SCVT (DAPT arm incidence, $16.06 \%)$. Again, this was performed using sample sizes as Fischer information could not be used due to too little information use $(-63.12 \%)$. In Figure 3, the $z$-score curve did not cross any boundary. This indicates that the observed effect was not conclusive, and more studies are required before any conclusion could be made. With an observed RRR of $-16.4 \%$, TSA was repeated with an RRR of $-20 \%$, with similar results that indicate that the current evidence is insufficient to confirm or rule out a $20 \%$ increase in SCVT risk in patients taking SAPT compared with those taking DAPT. TSA could also be repeated with an RRR of $-33.3 \%$, as shown in Figure 4, which shows that the $z$-score curve crossed the futility boundary. This leads to the conclusion that the existing evidence is sufficient to show that SAPT use is not associated with an increase in SCVT risk of $33.3 \%$ or more compared with DAPT. Similar observations were made when the TSA was performed using event size instead.

Although the conclusions of TSA may be attractive, one must bear in mind the limitations of TSA. Most importantly, virtually all limitations of meta-analyses are applicable to TSA as well, because TSA makes use of data from metaanalyses. Any significant risk of bias, including publication bias, can affect TSA results significantly. Therefore, TSA is ideally performed on study data with minimal risk of bias. Nonetheless, most meta-analyses inevitably include studies with high risks of bias. To ameliorate this issue, it is possible to estimate effect sizes for TSA only from studies with low risks of bias. On a similar note, significant heterogeneity also affects the validity of TSA results-in the above TSA example, whereas the OAC was shown to be conclusively superior to SAPT or DAPT for preventing SCVT, the results should be interpreted with caution as significant heterogeneity was detected $\left(I^{2}=68 \%\right)$. More detailed analysis, including subgroup analyses or meta-regression, may be performed to delineate the underlying cause of such heterogeneity and refine the conclusion. An alternative would be to perform TSA on outcomes without significant heterogeneity, because the heterogeneity would need to be addressed before the evidence could be considered conclusive and definitive anyway.

As useful as TSA may be in preventing spurious conclusions, TSA have also been criticized for being too conservative. ${ }^{15}$ This relates to the RRR selected for analysis-choosing RRR values that are too conservative easily leads to results showing that existing evidence is insufficient to confirm or rule out the chosen level of RRR. Indeed, this is mostly arbitrary in most cases and relies heavily on the clinical expertise and experience of the author. As shown in the above example comparing the stroke rates in patients with and without SCVT, it would be almost meaningless to repeat the TSA with a RRR of $-20 \%$, which is a common prespecified RRR value used in TSA: the $z$-score curve does not cross any boundary when such a small RRR is used, and the resultant conclusion that the current evidence is insufficient to confirm or rule out a $20 \%$ increase in stroke risk with SCVT is unhelpful given the strong, conclusive evidence that there is at least a $100 \%$ increase in stroke risk. ${ }^{16}$ Such difference in TSA results may seem counterintuitive, but is simply a result of the previously mentioned principle that more data are required to conclusively demonstrate a small effect size compared with a large effect size. The chosen RRR therefore directly influences the results and conclusion of TSA, and clinical relevance and appropriateness of any chosen RRR must be considered when interpreting TSA.

\section{CONCLUSIONS}

TSA of the meta-analysis by Woldendorp and colleagues ${ }^{13}$ revealed that existing evidence is sufficient to conclude at least a $100 \%$ increase in risk of stroke in patients with SCVT compared with those with NLM, as well as that OAC use is associated with at least $20 \%$ reduction in SCVT risk compared with SAPT or DAPT use. However, existing evidence is insufficient to rule out or confirm a $20 \%$ increase in SCVT risk in patients who take SAPT, compared with those taking DAPT; this was despite the evidence being sufficient to rule out an increase in SCVT risk of $33.3 \%$ or more. Our analysis demonstrated the key utility of TSA, an evolving tool that may be expected to play increasingly important roles in meta-analyses. Clinicians should familiarize themselves with TSA, and note the limitations of TSA when interpreting its results. When used and interpreted appropriately, TSA can be a powerful tool capable of assessing the conclusiveness of meta-analytical findings.

\section{Conflict of Interest Statement}

The authors reported no conflicts of interest.

The Journal policy requires editors and reviewers to disclose conflicts of interest and to decline handling or reviewing manuscripts for which they may have a conflict of interest. The editors and reviewers of this article have no conflicts of interest.

\footnotetext{
References

1. Borm GF, Donders ART. Updating meta-analyses leads to larger type 1 errors than publication bias. J Clin Epidemiol. 2009;62:825-30.e10.
} 
2. Brok J, Thorlund K, Gluud C, Wetterslev J. Trial sequential analysis reveals insufficient information size and potentially false positive results in many meta-analyses. J Clin Epidemiol. 2008;61:763-9.

3. Brok J, Thorlund K, Wetterslev J, Gluud C. Apparently conclusive meta-analyses may be inconclusive - trial sequential analysis adjustment of random error risk due to repetitive testing of accumulating data in apparently conclusive neonatal meta-analyses. Int J Epidemiol. 2009;38:287-98.

4. Chan JSK, Kot TKM, Ng M, Harky A. Continuous infusion versus intermittent boluses of furosemide in acute heart failure: a systematic review and meta-analysis. J Card Fail. November 12, 2019 [Epub ahead of print].

5. Zhang X, Wu Q, Wei M, Ding Y, Gu C, Liu S, et al. Low-residual diet versus clear liquid diet for bowel preparation before colonoscopy: meta-analysis and trial sequential analysis of randomized controlled trials. Gastrointest Endosc. May 3, 2020 [Epub ahead of print].

6. Yokoyama C, Mihara T, Kashiwagi S, Koga M, Goto T. Effects of intravenous dextrose on preventing postoperative nausea and vomiting: a systematic review and meta-analysis with trial sequential analysis. PLoS One. 2020;15: e0231958.

7. Ge X, Wang W, Hou L, Yang K, Fa X. Inspiratory muscle training is associated with decreased postoperative pulmonary complications: evidence from randomized trials. J Thorac Cardiovasc Surg. 2018;156:1290-300.

8. Hristovska A-M, Duch P, Allingstrup M, Afshari A. Efficacy and safety of sugammadex versus neostigmine in reversing neuromuscular blockade in adults. Cochrane Database Syst Rev. 2017;8:CD012763.
9. Chan JSK, Lau DHH. Does lysing make life better? A trial sequential analysis. $J$ Neurol. 2020;267:1842-5.

10. Chan JSK. Colchicine prevents stroke in patients with coronary artery disease-a trial sequential analysis. Eur J Neurol. 2020;27:e28.

11. Chan JSK. The results are in: cardiovascular benefits of glucagon-like peptide-1 receptor agonists - a trial sequential analysis. Eur J Prev Cardiol. April 10, 2020 [Epub ahead of print].

12. Manogaran M, Yang SS. Data for beta-blockade in ACLS - a trial sequential analysis. Resuscitation. 2020;150:191-2.

13. Woldendorp K, Doyle MP, Black D, Ng M, Keech A, Grieve SM, et al. Subclinical valve thrombosis in transcatheter aortic valve implantation: a systematic review and meta-analysis. J Thorac Cardiovasc Surg. February 20, 2020 [Epub ahead of print].

14. Thorlund K, Engstrøm J, Wetterslev J, Brok J, Imberger G, Gluud C. User Manual for Trial Sequential Analysis (TSA). Copenhagen, Denmark: Copenhagen Trial Unit; 2017.

15. Wetterslev J, Jakobsen JC, Gluud C. Trial sequential analysis in systematic reviews with meta-analysis. BMC Med Res Methodol. 2017 $17: 39$.

16. Roshanov PS, Dennis BB, Pasic N, Garg AX, Walsh M. When is a meta-analysis conclusive? A guide to Trial Sequential Analysis with an example of remote ischemic preconditioning for renoprotection in patients undergoing cardiac surgery. Nephrol Dial Transplant. 2017;32(Suppl 2): ii23-30. 